\title{
Rotary Liner Hanger Bearing Roller Modeling and Modification Based on Solidworks Secondary Development
}

\author{
Weiqing $\mathrm{Li}^{*}$, Zhijun Niu and Jin Xu \\ School of Engineering and Technology \\ China University of Geosciences (Beijing) \\ Beijing, 10083, China \\ *Corresponding author
}

\begin{abstract}
The main failure form of the rotary liner hanger bearing is roller end breakage in the drilling engineering. Some modification methods are applied to solve this problem, however, the modification formula is very complex and calculation process need to be repeated, which may lead to calculating negligence, and lower the accuracy of modification. In this paper, one rotating liner hanger bearing roller modification system is designed by applying Solidworks API functions, macro and VBA programming environment based on Solidworks secondary development platform and improved roller modification theory of rotating liner hanger bearing roller. With analyzing the working condition parameters, designing the modeling program, and designing interface and the result display module, a parameterized modeling and rapid modification of tapered roller system is achieved. This system greatly improved the efficiency and accuracy of the modeling and modification of roller, which provides some convenience for the subsequent research, analysis and manufacturing.
\end{abstract}

Keywords-bearing roller; roller Modification; automatic modeling; Solidworks secondary development

\section{INTRODUCTION}

In recent years, China's oil and gas exploration obtains vigorous development and large quantities of oil and gas wells have been mined. Cementing is a very important part in the process of oil and gas well production because the cementing quality largely effects on the production of the wells [1]. Current rotary liner cementing technology is widely used at home and abroad, however rotary liner hanger bearing is a vulnerable part of the cementing tools. The bearing life currently used is not able to meet the need, which seriously affects cementing efficiency and quality.

One of the main causes that lead to this situation is the overweight load during well cementation which causes the hanger bearing roller to have a serious edge effect [2], which lead to the roller premature fragmentation and the bearing failure.

In order to solve this problem, W.Q. Li adopted the scheme of roller modification to reduce the stress concentration and increased life expectancy of the rotary liner hanger bearing [3]. Therefore, the roller modification modeling of rotating liner hanger bearing is based on the improved modification theory in this paper. However, the improved modification formula is very complex because they include many parameters, which lead to the calculation process complicated and repeated. It may cause the negligence and error of accumulation, which will reduce the accuracy of modification and lead expected modification effect to be difficult to obtain. After the modification parameters are calculated, they need to be imported into the modeling software to establish the model, and then imported the model into AWB simulation analysis environment. These processes are cumbersome, wasting time and error prone. In order to solve the above problem, this paper built a secondary development system where the modification formula and calculation variables will be programmed and combined with $3 \mathrm{D}$ modeling function so that the modification model is able to be built quickly and automatically [4].

\section{TOTAL DESIGN SCHEME OF THE SYSTEM}

\section{A. Development Platform and Methods}

The development platform is based on Solidworks 3D modeling module and VBA programming environment nested in Solidworks software.

The design method is that API function and macro recording function were used to program the roller modification formulas and calculation parameters. The model is able to be obtained automatically by second development program [5].

\section{B. Process Diagram of the Secondary Development System}

To compile the program of rotating liner hanger bearing roller automatic modification model, the overall thought of this paper is based on modifying model [6]. Firstly, a basic model was pre-established, where the relationship of parameters and the geometric constraints were defined. Secondly, the model was imported into ANSYS software and analyzed if the model is able to meet need [7]. Thirdly, according to the analyzing results, modification methods were chose and corresponding modification modeling parameters were input. After reconstruction, the new model is obtained according to the modification parameters. Figure 1 is the overall logical structure diagram. 


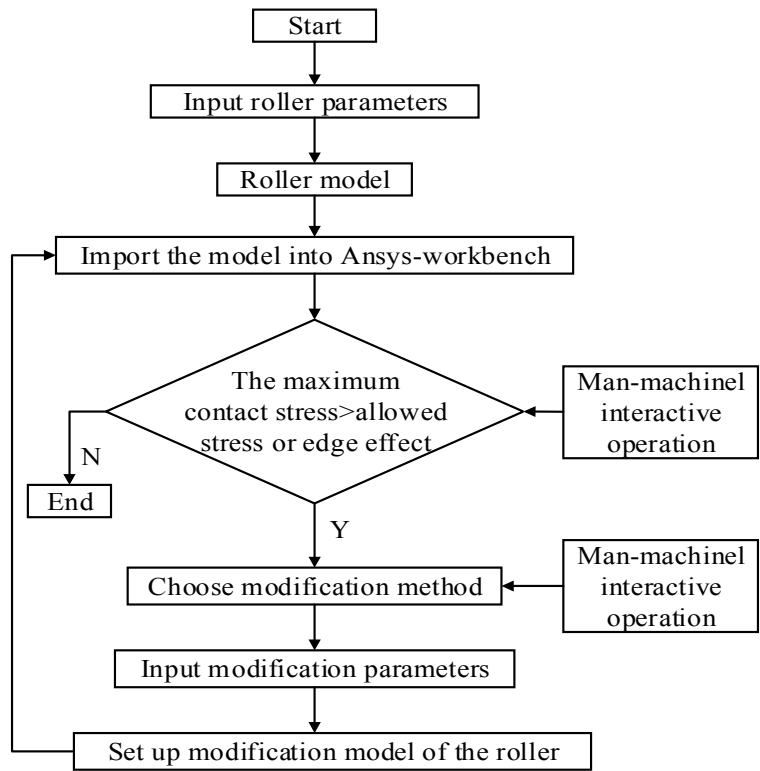

FIGURE I. PROCESS DIAGRAM OF THE SECONDARY DEVELOPMENT SYSTEM.

\section{PROGRAM IMPLEMENTATION AND OPERATING RESULTS}

Work related to the programming includes analyzing the working condition parameters, designing the modeling program, and designing interface and the result display module.

\section{A. Analysis of Modification Formula and Working Conditions Parameters}

The purpose of this system is to achieve automatic modeling of the roller based on the improved modification theory. (1) is the Lundberg logarithmic curve. (2) is the elastic approach which is included in modification equation. (3), (4) and (5) respectively are the expressions of the radius for intersecting, cut and convex modification curve. The parameters of deep-hole shape modification are i (hollow degree of deep hole) and $\mathrm{j}$ (modification depth) [8]. Therefore, working conditions parameters of roller modification includes a total of 12 parameters which need to be set in the system of automatic modification of the roller.

$$
\left\{\begin{array}{l}
\mathrm{Z}(\mathrm{y})=\mathrm{ALn}\left(1-\mathrm{By}^{2}\right) \quad-\frac{1}{2} \mathrm{~L}_{\text {eff }} \leq \mathrm{y} \leq \frac{1}{2} \mathrm{~L}_{\text {eff }} \\
\mathrm{A}=\left[0.06 \bullet\left(\frac{\mathrm{L}_{\text {eff }}}{\frac{1}{2} \mathrm{D}_{1}+\mathrm{D}_{2} / \cos \alpha}\right)-0.44\right] \cdot \frac{\mathrm{Q} \bullet \cos \alpha}{\mathrm{E}^{\prime} \mathrm{L}_{\text {eff }}} \\
\mathrm{B}=\left(1-0.6 \frac{\mathrm{P}_{0} \bullet \frac{1}{2} \mathrm{D}_{1}+\mathrm{D}_{1} / \cos \alpha}{\mathrm{L}_{\text {eff }} * \mathrm{E}^{\prime}}\right) \frac{4}{\mathrm{~L}_{\text {eff }}^{2}}
\end{array}\right.
$$

$$
\begin{gathered}
\delta=4.80 \bullet\left[\frac{1-\mathrm{v}_{1}^{2}}{\pi \mathrm{E}_{1}}+\frac{1-\mathrm{v}_{2}{ }^{2}}{\pi \mathrm{E}_{2}}\right]^{0.9} \cdot \frac{\mathrm{F}^{0.9}}{1_{\mathrm{eff}}^{0.74} \mathrm{D}_{\mathrm{we}}^{0.1}} \\
\mathrm{R}_{\mathrm{c}}=\frac{\delta}{4}+\frac{\mathrm{L}_{\mathrm{eff}}{ }^{2}}{4 \delta} \\
\mathrm{R}_{\mathrm{c}}=\sqrt{\left[\frac{\left(1-\mathrm{a}^{2}\right) \mathrm{L}_{\mathrm{eff}}{ }^{2}}{4 \delta}-\frac{\delta}{4}\right]^{2}+\frac{\mathrm{L}_{\mathrm{eff}}{ }^{2}}{4}} \\
\mathrm{R}_{\mathrm{c}}=\frac{\delta}{2}+\frac{(1-\mathrm{a})^{2} \cdot \mathrm{L}_{\mathrm{eff}}{ }^{2}}{4 \delta}
\end{gathered}
$$

Where, $\mathrm{F}$ is the vertical load of the roller generatrix. $\alpha$ is the half cone angle of the tapered roller. $E_{1}$ and $E_{2}$ respectively are the young's modulus of the roller material and ring material. $v_{1}$ and $v_{2}$ respectively are the poisson's ratio of the roller material and ring material. $\mathrm{D}_{1}$ and $\mathrm{D}_{2}$ respectively are the big end and small end diameter of the tapered roller. Leff is the length of the straight generatrix. $a$ is the contact width of roller and ring. $D_{w e}$ is the equivalent diameter of the tapered roller. $\mathrm{Z}$ is the modification crown of the Lundberg logarithmic generatrix.

In order to have a wider application space and be able to adapt to different working conditions, different geometry and different material parameters, the program in this paper reserved corresponding variable interfaces for 12 working condition parameters.

\section{B. Programming of Automatic Modeling for Roller}

Applying the thought of modifying model in Figure 1, considering the working conditions, and referring the method of Solidworks secondary development, this paper developed a rotary liner hanger bearing roller automatic modeling and modification system based on the Solidworks secondary development platform [9]. Taking the logarithmic modification generatrix as an example, the programming steps and contents are as follows.

\section{1) Pre-built roller model}

Combined with the improved modification theory, the model of the corresponding straight generatrix taper roller is built with modeling techniques, such as drawing sketches and defining corresponding parameters including the diameter of big end and small end of the roller, cone angle, effective contact length, straightness or determining the driving equation coefficient of the curve when generatrix modification method was chose [10]. When deep-hole modification method was chose, the coefficients of $i$ (hollow degree of deep hole) and $\mathrm{j}$ (modification depth) need to be defined.

The values of these parameters do not need to be accurate, but they must be defined when the model was built. These parameters need to be named according to certain rules and saved in the appropriate path.

2) Enter the program by the corresponding button Private Sub cmd

Logarithmic generatrix roller shape modeling_Click() 
3) Define basic variable and function object

Dim swApp As Object; Dim Part as Object

Dim boolstatus As Boolean

Dim longstatus As Long, longwarnings As Long

4) Establish application relationships with solidworks and documents

Set swApp $=\_$Application.SldWorks;

Set Part $=$ swApp.ActiveDoc

5) Open the file

API Set Part = swApp.OpenDoc6("path", 1, 0, “", longstatus, longwarnings)

Here, path parameter will show the file location and file name.

\section{6) Transform view}

This function is able to transform the view to the direction that is easy for user to observe, so that the user is convenient and intuitive to see the change of the modeling parameters.

Isometric observation view function: Part. Show Named View2 "*isometric drawing"

The whole screen view: Part.ViewZoomtofit2.

\section{7) Declare parameters of modification modeling}

Firstly, select the sketch that modification modeling parameters is at. Secondly, call the function that edits the sketch. Thirdly, declare the dimensions that edit the modification modeling. API are as follows.

Part. Extension. Select By ID2 ("Sketch 1", "SKETCH", 0, 0, 0, False, 0, Nothing, 0).

\section{Part. Edit Sketch}

boolstatus=Part. Extension. Select By ID2 ("D3@Sketch5@tangential_roller generatrix. SLDPRT","DIMENSION", 3.48133823588004E-02, 2.52116768219657E-02, 0, False, 0, Nothing, 0) .

Dim my Dimension As Object; Set my Dimension = Part. Parameter(“D3@Sketch 1").

8) Input the work condition parameters of the hanger bearing

12 work condition parameters are input by the manmachine interface. The parameters are transferred into the corresponding formula and calculated the result by background program.

Define 12 working condition parameters and declare the data types:

Dim F As Double; Dim $\alpha$ As Double; Dim D1 As Double; Dim D2 As Double;

Dim $\mu 1$ As Double; Dim $\mu 2$ As Double; Dim E1 As Double; Dim E2 As Double;
Dim Leff As Double; Dim a As Double; Dim $\pi$ As Double; $\pi=3.1415926$

Dim i as Double; Dim j as Double.

The values that user input man-machine interfaces are given to 12 work condition parameters:

$\mathrm{F}=\operatorname{CDbl}($ txtF.Text $) ; \alpha=\operatorname{CDbl}($ txt $\alpha \cdot$ Text $) ; \mathrm{D} 1=$ CDbl(txtD1.Text);

$\mathrm{D} 2=\operatorname{CDbl}(\operatorname{txtD} 2 . \mathrm{Text}) ; \mu 1=\operatorname{CDbl}(\operatorname{txt} \mu 1$. Text $) ; \mu 2=$ CDbl(txt $\mu 2 . T e x t)$;

$\mathrm{E} 1=\mathrm{CDbl}(\mathrm{txtE} 1$. Text $) ; \mathrm{E} 2=\mathrm{CDbl}(\mathrm{txtE} 2$. Text $) ;$ Leff $=$ CDbl(txtLeff.Text);

$a=\operatorname{CDbl}($ txta.Text);i= CDbl(txti.Text);j=CDbl(txtj.Text).

9) Substitute the working condition parameters into an improved formula (1) to calculate the theoretical modification value

$$
\begin{aligned}
& \mathrm{E}=\left(1-\mu 1^{\wedge} 2\right) / \mathrm{E} 1+\left(1-\mu 2^{\wedge} 2\right) / \mathrm{E} 2 \\
& \mathrm{Dwe}=0.5 *(\mathrm{D} 1+\mathrm{D} 2) / \operatorname{Cos}(0.5 * \alpha * \pi / 180) \\
& \mathrm{q}=\mathrm{F} * \operatorname{Cos}\left(0.5 * \alpha^{*} \pi / 180\right) \\
& \mathrm{f} 1=(-0.06 *(\text { Leff } / \text { Dwe })+0.44) * \pi \\
& \mathrm{c}=\mathrm{Sqr}(2 * \mathrm{q} * \text { Dwe } * \mathrm{E} /(\pi * \text { Leff })) \\
& \mathrm{Po}=2 * \mathrm{q} /(\pi * \mathrm{c} * \text { Leff }) \\
& \mathrm{A}=-\mathrm{f} 1 * \mathrm{q} * \mathrm{E} /(\pi * \text { Leff }) \\
& \mathrm{B}=(1-0.6 * \mathrm{E} * \text { Po } * \text { Dwe / Leff } * 4 /(\text { Leff }) \wedge 2 \\
& \mathrm{Z}(\mathrm{y})=\mathrm{CStr}(\mathrm{A}) \& * * \ln \left(1-" \& \mathrm{CStr}(\mathrm{B}) \& * * \mathrm{x}^{*} \mathrm{x}\right)
\end{aligned}
$$

10) Assign the calculation results to the modification parameters and rebuilt the modeling

$$
\begin{aligned}
& \text { TextBoxC.Text }=\operatorname{Val}(\mathrm{c}) \\
& \text { TextBoxD.Text }=\operatorname{Val}(\mathrm{d})
\end{aligned}
$$

Part.SketchManager.InsertSketch True.

\section{1) Transform view after modification}

Part.ShowNamedView2 "Around two isometric axis measurement", 9Part.ViewZoomtofit2.

\section{2) End program}

End Sub.

\section{Interface Design and Operating Results}

Some man-machine interfaces are design by using the toolbox of Solidworks VBA so that the users are convenient to input the work parameters and certain need [11]. The rules that design the interface are simple, operational and aesthetic.

The interface includes title bar, menu bar, auxiliary instructions graphics, user parameters input interface, the implementation buttons and etc. 


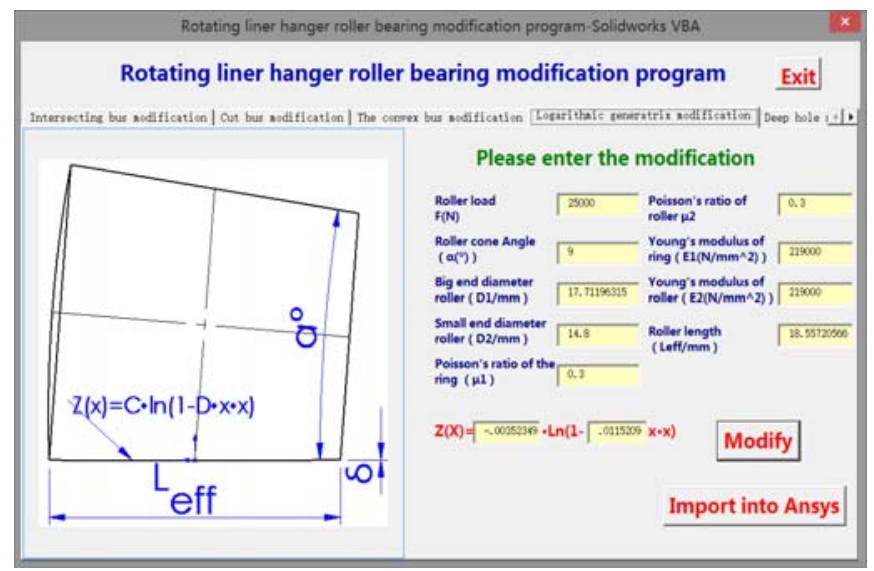

FIGURE II. INTERFACE DIAGRAM OF LOGARITHMIC GENERATRIX ROLLER MODIFICATION PROGRAM.

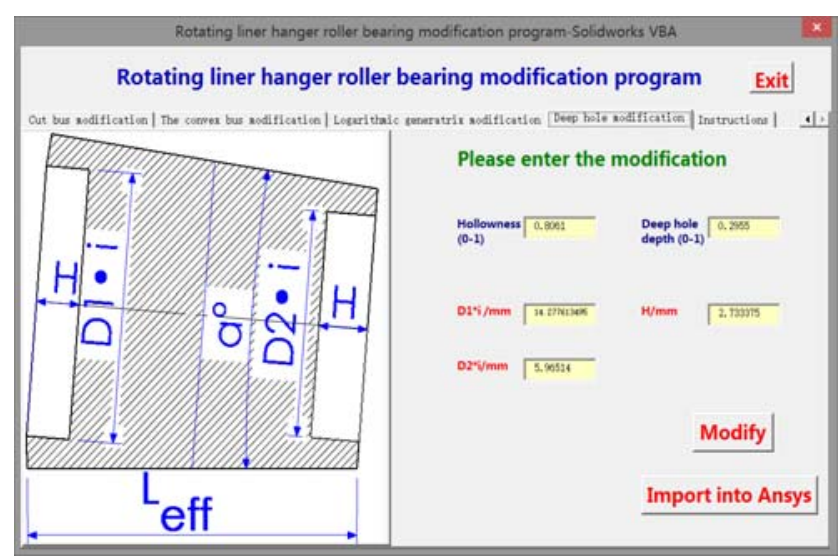

FIGURE III. INTERFACE DIAGRAM OF DEEP HOLE MODIFICATION PROGRAM

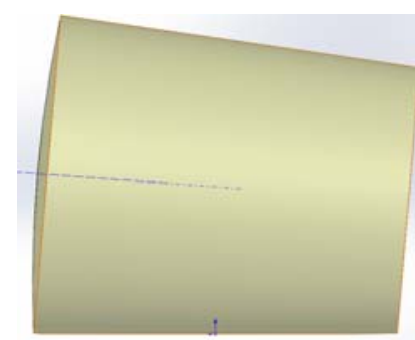

(a) Logarithmic roller

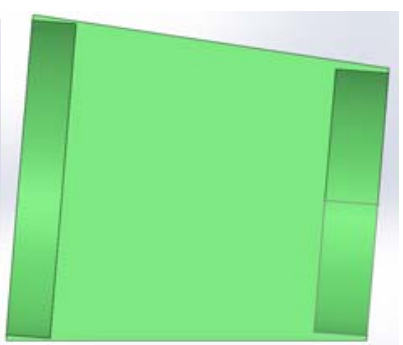

(b) Deep hole roller
FIGURE IV. THE RESULTS OF THE MODIFICATION AFTER MODELING

This paper also uses the toolbox "multi-page" command to differentiate the different roller modification methods. The corresponding working condition parameter input interface is set up in each page. At the interface of the input parameters, a note was done to specify the range of the input parameters. The corresponding executive command button and assist graphics were design to explain the subject.

Figure 2, Figure 3 and Figure 4 are respectively the interface of logarithmic modification, deep-hole modification and the result of modification modeling.

\section{CONCLUSION}

In this paper, one rotating liner hanger bearing roller modification system is designed by applying Solidworks API functions, macro and VBA programming environment. This system solved the problem in the roller modification such as multiple parameters, complex calculation, error-prone and low calculation efficiency. This system also solved the problem of artificial modeling inaccuracy and modeling process complication because the user can set up a corresponding modification type to meet the need of best modification effect model of roller by input the working parameters. This program provides some convenience for the subsequent research, analysis and manufacturing.

\section{ACKNOWLEDGMENT}

This project was supported by the Fundamental Research Funds for the Central Universities (265201304). This project also thanks Luoyang Bearing Science \& Technology Co., Ltd who provide us with test data so that we have the reference of the simulation research.

\section{REFERENCES}

[1] L.G. WU. "Development Status of Liner Cementing and Liner Completion Technology in China," [J]. Oil Field Equipment, 2013, (10): $2-56$.

[2] Weiqinf Li, Jianchong Wang. "Analysis of Thermo-Mechanical Coupling Characteristics and Structural Design of Rotary Liner Hanger Bearing in Ultra-Deep Well Drilling, ”. Proceedings of IMETI 2014. P33-39, (Orlando Florida, USA), July 15-18, 2014.

[3] W.Q. Li, Z.J. Niu and G.S. Chen. "Research on the Modification Technology for Tapered Roller Bearing of Rotary Liner Hanger in UltraDeep Well Drilling," International Conference on Mechanical and Automation Engineering, (Hong Kong), May 30-31, 2015. , in press.

[4] Solidworks company, Xiuzi Ye, Chaoqun Chen. "Solidworks Advanced Tutorial: Secondary development and API" [M]. Beijing: China Machine Press, 2009.

[5] J.S. Yin, B.Y SHENG. "Study on Product Design Expert System Based

[6] on the Secondary Development of SolidWorks," [J]. Chanical Engineering \& Automation, 2007, 5(3):61-63.

[7] X.T. Yan. "Parameterized Modeling of Parts Based on SolidWorks," [J]. Advanced Materials Research, 2011, (346):394-397.

[8] Weiwei Tu, Lixiang Zhang, Jun Sun. "Centrifugal Pump Volute Parametric Design Based on SolidWorks," [J]. Advanced Materials Research, Vols. 791-793 (2013): 714-717.

[9] Z.J. Niu. "Tapered Roller Modification Theory Research and Performance Optimization of Rotary Liner Hanger Bearing," [D]. Beijing: China University of Geosciences (Beijing), 2015.

[10] Li Sheng, Liangyou Zhang, Lixin Xie. "Analysis and discussion of precision sketch on secondary development of SolidWorks," [J]. Modern Manufacturing Engineering. 2014, (4): 68-71.

[11] Chang Ping Li; Jian Hua Rao; Lin Hu. "Solidworks Secondary Development System of DTH Bits," [J]. Advanced Materials Research, 2013 (753-755): 1065-1068.

[12] X M Li, W H Lu, X Ning, G C Jin,T C Yu. "A Rapid Design System for the Transmission System of Mounter Worktable Based on Second Development of SolidWorks," [J]. Advanced Materials Research, 2012, (605-607):592-595. 